TAIWANESE JOURNAL OF MATHEMATICS

Vol. 6, No. 2, pp. 157-174, June 2002

This paper is available online at http://www.math.nthu.edu.tw/tjm/

\title{
CONCAVITY, QUASICONCAVITY, AND QUASILINEAR ELLIPTIC EQUATIONS
}

\author{
John McCuan
}

\begin{abstract}
Quasiconcavity, the condition that the level sets of a positive graph are convex, is known to hold for solutions of certain semilinear equations. We survey some techniques that can be used to show quasiconcavity for solutions of quasilinear elliptic equations with form similar to the equation of constant mean curvature.
\end{abstract}

\section{INTRODUCTION}

A calculus student should recognize that a condition like

$$
\frac{\partial^{2} u}{\partial x^{2}}+\frac{\partial^{2} u}{\partial y^{2}}<0
$$

does not imply $\operatorname{graph}(u)$ is concave (down). Nevertheless, there are appropriate additional conditions that one can impose which ensure upwardly "bulging" graphs - as intuition might suggest - for solutions of elliptic equations that satisfy (1). One side condition that is almost universally imposed in the literature is a constant Dirichlet boundary condition on a convex domain. Accordingly, unless explicitly stated to the contrary, we will assume any function called $u$ is constant on the boundary of a convex domain called $\Omega$.

The literature, furthermore, focuses attention largely on solutions of semilinear equations $\Delta u=f(u)$ and imposes the additional side condition $u>0$ in $\Omega$ with $u_{\partial \Omega \Omega} \equiv 0$. In this case, the appropriate notion of bulging, it seems, is power concavity (i.e., that for some power $p$, one has $u^{p}$ is concave - or convex if $p<0$ ). As a matter of terminology, such a graph is also said to be $p$-concave. This direction is

Received August 20, 2001; revised September 20, 2001.

Communicated by Robert Finn.

2000 Mathematics Subject Classification: Primary 76B45, 76D45, 53A10; Secondary 49Q05, 53C42, 76E10.

Key words and phrases: constant mean curvature, quasiconcavity. 
introduced in the seminal paper of Brascamp and Lieb [2], and further developments may be found in [6].

Our interest is in solutions of quasilinear equations of the form

$$
\sum a_{i j}(D u) D_{i} D_{j} u=-k<0,
$$

where the coefficient matrix $a=\left(a_{i j}\right)>0$ (i.e., is positive definite) and $k$ is constant. According to the comparison principle [5], one always has in this case, $u \geq u_{\mid \partial \Omega}$, and the (constant) value of $u$ on $\partial \Omega$, which plays no role in the equation, may be prescribed. The form of the operator itself though, as we hope to indicate below, is not well suited to power concavity.

The weakest form of power concavity is, nevertheless, conjectured to hold for solutions of (2). This weakest form, associated to the exponent $p=-\infty$, is called quasiconcavity and is precisely the condition that the level sets $\Omega_{c}=\{x \in \Omega$ : $u(x) \geq c\}$ are convex.

Conjecture. All solutions of (2) with constant boundary values are quasiconcave.

The conjecture holds in the constant coefficient case; this assertion follows easily from the techniques presented in this paper, though our primary interest is in cases with nontrivial gradient dependence. Furthermore, if $\Omega$ is a ball, it can be shown that all solutions are concave. See the appendix to this paper.

The best known example of an equation of the form (2) is the equation of constant mean curvature (CMC):

$$
\operatorname{div}\left(\frac{D u}{\sqrt{1+|D u|^{2}}}\right)=2 H .
$$

Sakaguchi [15] has shown that the conjecture holds for this equation when $|H|$ is small enough. Related results, may be found in [3] where it is shown that any regions of negative Gauss curvature must be connected to $\partial \Omega$ and in [13] where it is shown, for example, that small solutions (i.e., for $|H|$ small) are concave when the domain satisfies additional assumptions. Finally, it should be noted that the present paper is primarily motivated by consideration of this particular equation.

The special divergence form and variational properties of (3) were used in most of the papers mentioned above. We follow a different course in this paper and give an exposition of two approaches that rely only on the comparison principle. The first approach is made possible by recent work on fully nonlinear equations [1]. In particular, this direction promises application to the more general class of equations (2) and offers a framework in which one can, perhaps, organize and compare the "strength" of various concavity maximum principle arguments which we describe 
by example in the following Section 1. In Section 2 we give some new results for certain equations of the form (2). In Section 3 we describe some of the difficulties encountered when the same techniques are applied to equation (3). In the final section, we describe a second approach, which suggests a more general notion of bulging (than quasiconcavity). This notion makes sense, in particular, for parametric surfaces.

\section{Concavity Maximum Principles}

If a graph is power concave for any power $p$, then it is quasiconcave (because the level set $\Omega_{c}=\{x: u(x) \geq c\}$ is equal to some level set of $\phi(u)=u^{p}$ ). More generally, as noted by many authors, given any monotone function $\phi=\phi(u)$, the concavity of $v=\phi(u)$ implies the quasiconcavity of $u$. We are unaware of any example in the literature, however, where the function $\phi$ is something other than a power.

Having chosen $\phi$, one wishes to show that the graph of $v=\phi(u)$ is concave. A concavity maximum principle argument can be viewed as doing this by showing the concave envelope $v^{*}$ of $v$ is a subsolution of an elliptic equation satisfied by $v$ and then applying a maximum principle to conclude $v^{*} \leq v$. Since $v^{*} \geq v$ by definition (see below), one has that $v$ is concave.

This strategy was first applied successfully by Korevaar [9] (though he did not describe it in this way), and using it, he was able to give an alternative proof [10] of the result of Brascamp and Lieb mentioned above. Kennington [7] later generalized the basic argument of Korevaar and was able to treat the Poisson equation $\Delta u=-k$. In their recent paper [1], Alvarez, Lazry and Lions prove all of Korevaar's and Kennington's results from a more general perspective, and we begin our exposition from their point of view.

Accordingly, let us assume that $v$ is a smooth solution of

$$
F\left(v, D v, D^{2} v\right)=0
$$

where $F$ is defined on $\mathbb{R} \times \mathbb{R}^{n} \times M^{n}$ (the last factor being $n \times n$ matrices), smooth on the closure of $v(\Omega) \times D v(\Omega) \times D^{2} v(\Omega)$, and elliptic in the sense that if $(z, p) \in v(\Omega) \times D v(\Omega)$ is fixed and $A-B$ is positive semidefinite (i.e., $A \geq B$ ), then

$$
F(z, p, A) \geq F(z, p, B) .
$$

We furthermore assume that $v$ satisfies the Neumann condition $\partial v / \partial n=-\infty$ with respect to the outward normal on $\partial \Omega$. The Neumann condition may also hold for (4) in the generalized sense that for (some points) $x \in \partial \Omega$ one has $v(x)=-\infty$. 
The concave envelope $v^{*}$ of $v$ is given by

$$
v^{*}(x)=\max \left\{\lambda_{1} v\left(x_{1}\right)+\cdots+\lambda_{k} v\left(x_{k}\right)\right\}
$$

where the maximum is taken over all $\lambda_{1}, \ldots, \lambda_{k} \geq 0$ and all $x_{1}, \ldots, x_{k} \in \Omega$ such that $\lambda_{1}+\cdots+\lambda_{k}=1$ and $x=\lambda_{1} x_{1}+\cdots+\lambda_{k} x_{k}$. At a point $x \in \Omega$ for which $v^{*}(x)>v(x)$, one has that $D v\left(x_{1}\right)=\cdots=D v\left(x_{k}\right)$ and $D^{2} v\left(x_{j}\right) \leq 0$ for each $j$. The key estimate for the applications we describe below is

Lemma 1.1. If $w$ is a smooth function satisfying $w \geq v^{*}$ on $\Omega$ with equality at $x$, then

$$
D^{2} w(x) \geq \lim _{\delta \rightarrow 0}\left[\sum \lambda_{j}\left(D^{2} v\left(x_{j}\right)-\delta I\right)^{-1}\right]^{-1} .
$$

Notes. The presence of a smooth function $w$ standing in for a merely continuous function $v^{*}$ is typical of the theory of viscosity solutions. One should think of (6) as an estimate for " $D^{2} v^{*}$."

Note also that the nonpositivity of $D^{2} v\left(x_{j}\right)$ implies the matrix $D^{2} v\left(x_{j}\right)-\delta I$ is invertible. Moreover, the limiting term in (6) is increasing in $\delta$ (with respect to the usual partial order on matrices) so that the limit exists. Here also, the presence of the limit and the $\delta I$ is a technicality, and one should think of the estimate without them.

An estimate like (6) is a natural point of departure for showing that $v^{*}$ constitutes a subsolution in the viscosity sense, which means that for any $x \in \Omega$ and any smooth $w \geq v^{*}$ on $\Omega$ (with equality at $x$ as before) one has

$$
F\left(v^{*}(x), D w(x), D^{2} w(x)\right) \geq 0 .
$$

We mention, for the moment, that if (7) can be established, the viscosity theory provides a nice array of comparison principles with which one can often conclude $v^{*} \leq v$. We come back to this point below, but first we illustrate the general use of (6). Applying the ellipticity, we have

$$
\begin{aligned}
F & \left(v^{*}(x), D w(x), D^{2} w(x)\right) \\
& \geq F\left(v^{*}(x), D w(x), \lim _{\delta \rightarrow 0}\left[\sum \lambda_{j}\left(D^{2} v\left(x_{j}\right)-\delta I\right)^{-1}\right]^{-1}\right) \\
& =\lim _{\delta \rightarrow 0} F\left(\sum \lambda_{j} v\left(x_{j}\right), D v\left(x_{j}\right),\left[\sum \lambda_{j}\left(D^{2} v\left(x_{j}\right)-\delta I\right)^{-1}\right]^{-1}\right) .
\end{aligned}
$$

As pointed out by Alvarez, Lazry and Lions, the form of the limiting term in the Hessian slot suggests a desirable structure condition on $F$, namely, for every fixed $p$,

$$
(z, A) \mapsto F\left(z, p, A^{-1}\right) \quad \text { is concave. }
$$


More precisely, the concavity is required on $v(\Omega) \times \mathrm{Sym}^{-}$where $\mathrm{Sym}^{-}$denotes the negative definite, symmetric $n \times n$ matrices. Given this condition, one obtains

$$
\begin{aligned}
F & \left(v^{*}(x), D w(x), D^{2} w(x)\right) \\
& \geq \lim _{\delta \rightarrow 0} \sum \lambda_{j} F\left(v\left(x_{j}\right), D v\left(x_{j}\right),\left[\left(D^{2} v\left(x_{j}\right)-\delta I\right)^{-1}\right]^{-1}\right) \\
& =\lim _{\delta \rightarrow 0} \sum \lambda_{j} F\left(v\left(x_{j}\right), D v\left(x_{j}\right), D^{2} v\left(x_{j}\right)-\delta I\right) \\
& =\sum \lambda_{j} F\left(v\left(x_{j}\right), D v\left(x_{j}\right), D^{2} v\left(x_{j}\right)\right) .
\end{aligned}
$$

Since $v$ is a solution, this establishes (7). Unfortunately, we are often not given (8), as indicated in the following example.

Example 1.1 (Kennington's theorem). Assume $\Delta u+2 k=0$ with $k$ a positive constant. Let $v^{2}=u$. It follows that

$$
\left\{\begin{array}{l}
v \Delta v+|D v|^{2}+k=0 \quad \text { on } \Omega, \\
\frac{\partial v}{\partial n}=-\infty \quad \text { on } \partial \Omega .
\end{array}\right.
$$

(The boundary condition follows from the Hopf boundary point lemma.) We set $F\left(v, D v, D^{2} v\right)=v \operatorname{tr}\left[D^{2} v\right]+|D v|^{2}+k$ and since $v \geq 0$ ellipticity holds. We have then from Lemma 1.1,

$$
\begin{aligned}
& v^{*}(x) \Delta w(x)+|D w(x)|^{2}+k \\
& \geq \lim _{\delta \rightarrow 0} v^{*}(x) \operatorname{tr}\left[\sum \lambda_{j}\left(D^{2} v\left(x_{j}\right)-\delta I\right)^{-1}\right]^{-1}+|D w(x)|^{2}+k .
\end{aligned}
$$

It is easily verified that $(v, A) \mapsto v \operatorname{tr} A^{-1}$ is not concave on $[0, \infty) \times \mathrm{Sym}^{-}$. In fact, restricting to the span of the identity (or equivalently considering the case $n=1$ ), we can think of $A=\alpha$ as a scalar, and the relevant $2 \times 2$ Hessian has determinant

$$
\operatorname{det}\left(\begin{array}{cc}
0 & -1 / \alpha^{2} \\
-1 / \alpha^{2} & 2 v / \alpha^{3}
\end{array}\right)<0
$$

This means the eigenvalues have different signs and, hence, the Hessian is not nonpositive.

Consider, however, the mapping $(v, A) \mapsto v^{2} \operatorname{tr} A^{-1}$. We will show below (Lemma 1.3) that this mapping is concave. Therefore,

$$
\left[v^{*}(x)\right]^{2} \operatorname{tr}\left[\sum \lambda_{j}\left(D^{2} v\left(x_{j}\right)-\delta I\right)^{-1}\right]^{-1} \geq \sum \lambda_{j}\left[v\left(x_{j}\right)\right]^{2}\left(D^{2} v\left(x_{j}\right)-\delta I\right) .
$$


Multiplying both sides of (9) by $v^{*}(x)$, substituting the last inequality, and evaluating the limit, we have

$$
\begin{aligned}
& {\left[v^{*}(x)\right]^{2} \Delta w(x)+\left(|D w(x)|^{2}+k\right) v^{*}(x)} \\
& \geq \sum \lambda_{j}\left[v\left(x_{j}\right)\right]^{2} \Delta v\left(x_{j}\right)+\left(|D w(x)|^{2}+k\right) v^{*}(x) .
\end{aligned}
$$

Since $v^{*}(x)=\sum \lambda_{j} v\left(x_{j}\right)$ and $v$ is a solution, we get

$$
\left[v^{*}(x)\right]^{2} \Delta w(x)+\left(|D w(x)|^{2}+k\right) v^{*}(x) \geq 0 .
$$

The extra factor $v^{*}(x)$ may now be harmlessly canceled, and one sees that $v^{*}$ is a subsolution.

We will generalize several aspects of this example to equations of the form (2). The general consideration of comparison principle in that discussion will imply $v^{*}=v$.

Looking back at Example 1.1 and forward to more general operators, one would like to know when the operator

$$
F\left(v, D v, D^{2} v\right)=\psi^{\prime} \operatorname{tr}\left[a\left(\psi^{\prime} D v\right) D^{2} v\right]+\psi^{\prime \prime} \operatorname{tr}\left[a\left(\psi^{\prime} D v\right) D v D v^{T}\right]+k
$$

satisfies (8) where $\psi=\phi^{-1}$ is the inverse of a monotone rearrangement $\phi$. (We obtain (10) by substituting $u=\psi(v)$ in (2).)

In Example 1.1, $\psi^{\prime}(v)=v$, and the first term $v \Delta v$ failed to have the required concavity. This failure occurs quite generally for $1 / 2$-power rearrangement (which turns out to be a useful fact to know).

Lemma 1.2. The mapping $(v, A) \mapsto v \Phi(A)$ is never concave on $[0, \infty) \times \mathrm{Sym}^{-}$ unless $\Phi$ is constant.

Proof. Assume by way of contradiction that the mapping is concave and $\partial \Phi / \partial \alpha_{i j}$ is nonzero at some negative definite $A=\left(\alpha_{i j}\right)$. Let $E_{i j}$ be the matrix with 1 at entry $i j$ and 0 elsewhere. For $\alpha \in \mathbb{R}$ small, $A+\alpha E_{i j}$ is negative, so the restriction $(v, \alpha) \mapsto v \Phi\left(A+\alpha E_{i j}\right)$ is concave. The Hessian of this map at $\alpha=0$ is

$$
\left(\begin{array}{cc}
0 & \left\langle D \Phi(A), E_{i j}\right\rangle \\
\left\langle D \Phi(A), E_{i j}\right\rangle & \left\langle D^{2} \Phi(A) E_{i j}, E_{i j}\right\rangle
\end{array}\right) .
$$

This matrix has determinant

$$
-\left[\frac{\partial \Phi}{\partial \alpha_{i j}}(A)\right]^{2}<0
$$


which is a contradiction.

One would like to understand the structure of all operators satisfying (8). A modest result in this direction is suggested by the appendix to [1]:

Lemma 1.3. If $\mu:[0, \infty) \rightarrow[0, \infty)$ is a function for which $\sqrt{\mu}$ is convex, then $(v, A) \mapsto \mu \operatorname{tr}\left(a A^{-1}\right)$ is concave on $[0, \infty) \times \mathrm{Sym}^{-}$where a is a constant positive definite coefficient matrix.

We obtain Lemma 1.3 as a corollary of a somewhat technical generalization that we use for certain applications.

Lemma 1.4. Let $a=a(v, p)$ be a positive definite matrix with the following property:

If $N$ is orthogonal, $C$ is symmetric, and $b$ denotes a diagonal element of $N C a C N^{T}$, then for fixed $p \in \mathbb{R}^{n}, \sqrt{b}$ is a convex function of $v$.

Then for fixed $p$, the map $(v, A) \mapsto \operatorname{tr}\left[a(v, p) A^{-1}\right]$ is concave on $[0, \infty) \times \mathrm{Sym}^{-}$. Note that taking $a=\mu a_{0}$ where $a_{0}$ is a constant matrix, we get Lemma 1.3 as a corollary.

Proof of Lemma 1.4. First note that $b \geq 0$ so that $\sqrt{b}$ makes sense. In fact,

$$
b=b_{j j}=\left\langle N C a C N^{T} e_{j}, e_{j}\right\rangle=\left\langle a C N^{T} e_{j}, C N^{T} e_{j}\right\rangle \geq 0
$$

where $e_{1}, \ldots, e_{n}$ denote the standard basis vectors of $\mathbb{R}^{n}$.

Next, note that the convexity of $\sqrt{b}$ implies the joint concavity of $(v, \alpha) \mapsto b / \alpha$ on $[0, \infty) \times(-\infty, 0)$. (This can be used as an alternative hypothesis in the lemma.) To see that this joint concavity is an equivalent condition, compute the Hessian

$$
S=\left(\begin{array}{cc}
b^{\prime \prime} / \alpha & -b^{\prime} / \alpha^{2} \\
-b^{\prime} / \alpha^{2} & 2 b / \alpha^{3}
\end{array}\right) .
$$

Since $(\sqrt{b})^{\prime \prime}=\left(2 b b^{\prime \prime}-b^{\prime 2}\right) /\left(4 b^{3 / 2}\right) \geq 0$ and $b \geq 0$, we see that $b$ and $b^{\prime \prime}$ are nonnegative and, hence, $\operatorname{tr} S$ is nonpositive. Furthermore, $\operatorname{det} S \geq 0$, so $(v, \alpha) \mapsto$ $b / \alpha$ is concave as stated. Here we wrote $b(v)=b(v, p)$ since the $p$ dependence is relatively unimportant.

For the main assertion, we need to show

$$
\begin{aligned}
& \operatorname{tr}\left[a((1-\lambda) v+\lambda w)[(1-\lambda) A+\lambda B]^{-1}\right] \\
& \quad \geq(1-\lambda) \operatorname{tr}\left[a(v) A^{-1}\right]+\lambda \operatorname{tr}\left[a(w) B^{-1}\right] .
\end{aligned}
$$


Since $A^{-1}$ is symmetric and negative definite, there is a symmetric matrix $C$ such that $A^{-1}=-C^{2}$. If we replace $A$ by $-C^{-2}$ and write $\tilde{B}=C B C$, we see that (11) follows if

$$
\begin{array}{r}
\operatorname{tr}\left[a((1-\lambda) v+\lambda w) C[-(1-\lambda) I+\lambda \tilde{B}]^{-1} C\right] \\
\geq(1-\lambda) \operatorname{tr}\left[-a(v) C^{2}\right]+\lambda \operatorname{tr}\left[a(w) C \tilde{B}^{-1} C\right]
\end{array}
$$

for every negative symmetric $\tilde{B}$. On the other hand, $\tilde{B}$ can be diagonalized by an orthogonal matrix. Writing $\tilde{B}=N^{T} D N$, we need to show

$$
\begin{array}{r}
\operatorname{tr}\left[a((1-\lambda) v+\lambda w) C N^{T}[-(1-\lambda) I+\lambda D]^{-1} N C\right] \\
\geq(1-\lambda) \operatorname{tr}\left[-a(v) C^{2}\right]+\lambda \operatorname{tr}\left[a(w) C N^{T} D^{-1} N C\right] .
\end{array}
$$

That is,

$$
\begin{gathered}
\operatorname{tr}\left[b((1-\lambda) v+\lambda w)[-(1-\lambda) I+\lambda D]^{-1}\right] \\
\quad \geq(1-\lambda) \operatorname{tr}[-b(v) I]+\lambda \operatorname{tr}\left[b(w) D^{-1}\right]
\end{gathered}
$$

where $b=N C a C N^{T}$. Since the inverted matrices are now diagonal, we want

$$
\sum \frac{b_{j j}((1-\lambda) v+\lambda w)}{-(1-\lambda)+\lambda D_{j j}} \geq \sum(1-\lambda) \frac{b_{j j}(v)}{-1}+\lambda \frac{b_{j j}(w)}{D_{j j}} .
$$

Since by assumption $b_{j j} / \alpha$ is concave, we have that the inequality just stated holds termwise (without the summations), so the lemma follows.

At least Lemma 1.4 provides a criterion we can use in trying to analyze (10) or some "equivalent" operator. More precisely we formalize the procedure used in Example 1.1:

Definition 1. Given a subsolution $v$ of $F\left(v, D v, D^{2} v\right)=0$ where $F$ is given in (10) and $\phi$ is a rearrangement of $v(\Omega)$, an elliptic operator $\tilde{F}=\tilde{F}\left(v, D v, D^{2} v\right)$ is called $\phi$-adequate (or $\psi$-adequate if we wish to stress specification of the inverse) if

(i) A continuous function $w$ is a subsolution of $\tilde{F}\left(w, D w, D^{2} w\right)=0$ if and only if $w$ is a subsolution of $F\left(w, D w, D^{2} w\right)=0$.

(ii) $\tilde{F}$ satisfies the concavity condition (8).

In Example 1.1 the $\psi$-adequate operator was $\tilde{F}=v^{2} \Delta v+\left(|D v|^{2}+k\right) v$.

We will now allow the strong comparison properties of (2) to guide our discussion in two respects. The first involves a simplification achieved by restricting attention to (smooth) increasing rearrangements $\phi:[0, \infty) \rightarrow[0, \infty)$ for which $\phi(0)=0$ and $\phi^{\prime}(0)=\infty$ (or equivalently, $\psi(0)=0=\psi^{\prime}(0)$ ). The Hopf boundary 
point lemma then implies $\partial v / \partial n=-\infty$ on $\partial \Omega$. The second fact that simplifies our discussion is the following:

Lemma 1.5. If $v^{*}$ is a (viscosity) subsolution of $F\left(v, D v, D^{2} v\right)=0$ with $F$ given in $(10)$, then $u^{(*)}=\psi\left(v^{*}\right)$ is a subsolution of $\operatorname{tr}\left[a(D u) D^{2} u\right]+k=0$, which clearly admits a comparison principle. Consequently, u is quasiconcave.

Proof. If $w$ is a smooth function with $w \geq u^{(*)}$ and $w(x)=u^{(*)}(x)$ at some $x \in \Omega$, then $w=\psi\left(w_{0}\right)$ for some smooth $w_{0} \geq v^{*}$ and $w_{0}(x)=v^{*}(x)$. It follows that

$$
F\left(w_{0}(x), D w_{0}(x), D^{2} w_{0}(x)\right) \geq 0 .
$$

The left side of this inequality is exactly $\operatorname{tr}\left[a(D w(x)) D^{2} w(x)\right]+k$. Therefore, $u^{(*)}$ is a subsolution as claimed. If follows from Theorem 3.3 of [4] that $u^{(*)} \leq u$, and consequently, $v^{*} \equiv v$.

The discussion of this section, in summary, reduces verification of the main Conjecture to finding an appropriate rearrangement $\psi:[0, \infty) \rightarrow[0, \infty)$ such that $\psi(0)=0=\psi^{\prime}(0)$ and

$$
F(v, p, A)=\psi^{\prime} \operatorname{tr}\left[a\left(\psi^{\prime} p\right) A\right]+\psi^{\prime \prime} \operatorname{tr}\left[a\left(\psi^{\prime} p\right) p p^{T}\right]+k
$$

admits a $\psi$-adequate operator.

We note furthermore that any operator

$$
\tilde{F}=Q\left(\operatorname{tr}\left[\psi^{\prime} a\left(\psi^{\prime} p\right) A\right]+R\right)+Q\left(\operatorname{tr}\left[\psi^{\prime \prime} a\left(\psi^{\prime} p\right) p p^{T}\right]+k-R\right),
$$

where $R$ is any function of $v$ and $Q$ is any positive increasing function (which may also have an auxiliary $v$ dependence, satisfies condition (i) in the definition of $\psi$-adequate. Thus, within this (very general) family of operators, concavity is the only condition to be verified.

\section{Two EXAMPLES}

In this section we attempt to verify the hypotheses outlined in the last section (i.e., we try to find a $\psi$ and a $\psi$-adequate operator $\tilde{F}$ ) for the equations

$$
\left(1+|D u|^{2}\right)^{3 / 2} \operatorname{div}\left(\frac{D u}{\sqrt{1+|D u|^{2}}}\right)=-2 k
$$

and

$$
\frac{\Delta u}{\left(1+|D u|^{2}\right)^{3 / 2}}=-k
$$


It will be noted immediately that the mean curvature operator appears in (12), though the factor $\left(1+|D u|^{2}\right)^{3 / 2}$ eliminates the scaling that makes mean curvature a curvature. Equation (13) retains the appropriate scaling, but the numerator takes the degenerate form of the standard Laplacian, so that (13) only gives curvature in dimension one.

We begin by establishing

Theorem 2.1. Any solution u of (12) with zero boundary values on a convex domain is quasiconcave.

Proof. Setting $u=\psi(v)$, the associated equation for $v$ is given by

$$
\left.\psi^{\prime} \sum_{i, j}\left[\delta_{i j}\left(1+\psi^{\prime 2}\right)|D v|^{2}\right)-\psi^{\prime 2} D_{i} v D_{j} v\right] D_{i} D_{j} v+\psi^{\prime \prime}|D v|^{2}+2 k=0,
$$

which we may write as

$$
\psi^{\prime} \operatorname{tr}\left[a D^{2} v\right]+\psi^{\prime \prime}|D v|^{2}+2 k=0
$$

where $a=I+\psi^{\prime 2}\left(|D v|^{2} I-D v D v^{\mathrm{T}}\right)$. In this case, we take $\psi(v)=v^{2}$ as in Example 1.1, and it is easy to see that the operator

$$
\tilde{F}=v^{2} \operatorname{tr}\left[a D^{2} v\right]+\left(|D v|^{2}+k\right) v
$$

is elliptic and satisfies condition (i) of Definition 1. To see that condition (ii) holds as well, we look at the diagonal elements of $N C a C N^{-1}$ and apply Lemma 1.4:

$$
\begin{aligned}
N C a C N^{-1} & =N C^{2} N^{\mathrm{T}}+4 v^{2}\left(|p|^{2} N C^{2} N^{\mathrm{T}}-N C p p^{\mathrm{T}} C N^{\mathrm{T}}\right) \\
& =\left(C N^{\mathrm{T}}\right)^{\mathrm{T}} C N^{\mathrm{T}}+4 v^{2}\left[|p|^{2}\left(C N^{\mathrm{T}}\right)^{\mathrm{T}} C N^{\mathrm{T}}-\left(p^{\mathrm{T}} C N^{\mathrm{T}}\right)^{\mathrm{T}} p^{\mathrm{T}} C N^{\mathrm{T}}\right] .
\end{aligned}
$$

The $i$-th diagonal element is therefore

$$
\begin{aligned}
b_{i i}= & \mid i \text {-th column of }\left.C N^{\mathrm{T}}\right|^{2}+4 v^{2}\left[|p|^{2} \mid i \text {-th column of }\left.C N^{\mathrm{T}}\right|^{2}\right. \\
& \left.-\left(i \text {-th entry of } p^{\mathrm{T}} C N^{\mathrm{T}}\right)^{2}\right] \\
= & \left|C N_{i}\right|^{2}+4 v^{2}\left(|p|^{2}\left|C N_{i}\right|^{2}-\left\langle p, C N_{i}\right\rangle^{2}\right)
\end{aligned}
$$

where $N_{i}$ is the $i$-th row of $N$. Therefore, by Schwarz's inequality $b_{i i}$ is of the form $P_{1}+P_{2} v^{2}$ where $P_{1}$ and $P_{2}$ are nonnegative numbers depending only on $p=D v$. It is easily checked that the square root of such an expression is convex in $v$ (and so is the same expression times $v^{2}$ ). By the discussion in the last section, this establishes quasiconcavity for solutions $u$ of (12). 
The initial operator associated to equation (13) is

$$
F\left(v, D v, D^{2} v\right)=\frac{\psi^{\prime} \Delta v+\psi^{\prime \prime}|D v|^{2}}{\left(1+\psi^{\prime 2}|D v|^{2}\right)^{3 / 2}}+k .
$$

After rearrangement of the equation, we obtain the auxiliary operator

$$
\tilde{F}=-\frac{1}{\Delta v}-\frac{\psi^{\prime}}{k\left(1+\psi^{\prime 2}|D v|^{2}\right)^{3 / 2}+\psi^{\prime \prime}|D v|^{2}} .
$$

A similar rearrangement may be found in [1] where it is shown that $\Phi(A)=$ $-1 / \operatorname{tr} A$ is elliptic and satisfies the concavity condition (8). It remains for us to choose $\psi$ so that the remainder of the expression is concave.

Ideally, our choice of $\psi$ would provide that condition (ii) of Definition 1 holds for all positive $v$ and $P=|D v|^{2}$. Since we do not know how to make such a choice, we use elliptic theory to restrict these values in a natural way. To be precise, the methods of [13] can be extended to obtain

Lemma 2.1. There are positive constants $K>0$ and $\Lambda>0$ depending on the domain $\Omega$ such that every solution of the zero Dirichlet boundary value problem for (13) with $k \leq K$ satisfies

$$
|u|=|\psi(v)| \leq \Lambda k \quad \text { and } \quad P \psi^{\prime 2} \leq \Lambda^{2} k^{2} .
$$

Thus, we redefine our aim to show convexity of the function

$$
f(v)=\frac{g}{P g^{\prime}+k\left(1+P g^{2}\right)^{3 / 2}},
$$

where $g=\psi^{\prime}$ and $P=|D v|^{2}$, for small enough $k$, where we understand from the bounds of the lemma that this will also force $v$ and $P g^{2}$ to be small. Given a choice of $\psi$ and the condition that $g(0)=\psi^{\prime}(0)=0$, we also obtain from (17) another bound for $g(v)$ independent of $P$ but depending on $\psi$.

Having made these observations, we calculate $f^{\prime \prime}$. The expression for $f^{\prime \prime}$, though not suitable for the printed page, turns out to yield the following information concerning an adequate choice of $g$ :

1. One must have $g^{\prime \prime}(v)$ positive when $v$ is small. (The sign of $f^{\prime \prime}$ is determined by $g^{\prime \prime}$ when $P=0$, which evidently occurs for arbitrarily small values of $v$.)

2. By considering small values of $v$ under the assumption that $P g^{2}$ is only bounded, one obtains the necessary conditions $g^{\prime}(0)=0$ and $g^{\prime \prime}(0)=+\infty$. (Boundedness is a reasonable apriori assumption since the exact relationship between the decay of $v$ and that of $P g^{2}$ depends on $\psi$ which we are trying to choose.) 
3. It will be convenient if $g^{\prime \prime \prime}$ is negative. (In the expression for $f^{\prime \prime}$, the terms involving $g^{\prime \prime \prime}$ all appear with a negative sign.)

Evidently, any positive multiple of $g(v)=v^{3 / 2}$ satisfies these requirements. Indeed, having made this choice, $P g^{2}=P v^{3}$, and one sees that the sign of $f^{\prime \prime}$ is determined by

$$
\begin{aligned}
& 3 k P v^{3}-156 k P^{2} v^{6}-240 k P^{3} v^{9}+6 k^{2} \sqrt{1+P v^{3}} v^{5 / 2} \\
& -162 k^{2} P v^{3} \sqrt{1+P v^{3}} v^{5 / 2}-72 k^{2} P^{2} v^{6} \sqrt{1+P v^{3}} v^{5 / 2} \\
& +96 k^{2} P^{3} v^{9} \sqrt{1+P v^{3}} v^{5 / 2} \geq 2 k P v^{3} \geq 0
\end{aligned}
$$

as long as $P v^{3}<1 /(4 \cdot 156), k<1 /(8 \cdot 162)$, and $v<1$. The $1 / 4$ in the required bound on $P v^{3}$ came from the fact that there were four negative terms to be absorbed; the extra factor of $1 / 2$ in the $k$ bound is used to cover the square root factor in the third negative term; two positive terms are thrown away.

We arrive then at the following result.

Theorem 2.2. Small solutions of (13) satisfying a zero Dirichlet boundary condition on a convex domain are 2/5-concave.

The small data result just stated differs significantly from those in [15] or [13]. The most obvious difference is that arguments like those in the papers mentioned require estimates like those in Lemma 2.1 for $u,|D u|$, and the second derivatives $\left|D^{2} u\right|$. Furthermore, the former results, being tied to the solution of a linear PDE, only give a conclusion for $u,|D u|$, and $\left|D^{2} u\right|$ small enough. The proof of Theorem 2.2, however, being based essentially on an algebraic equation, gives explicitly (in terms of the domain) how small $u$ and $|D u|$ must be for the conclusion to hold.

As a final note, we mention that Alvarez, Lazry, and Lions also show ellipticity and concavity for the more general operator $\Phi(A)=-1 / \operatorname{tr}[a A]$ where $a$ is any positive definite constant coefficient matrix. We could, thus, have replaced the Laplacian in equation (13) with a general constant coefficient operator. Another consequence is that repeating the reasoning above for the special case of equation (2) in which the coefficients are constant, one sees that (16) becomes

$$
\tilde{F}=-\frac{1}{\operatorname{tr}\left[a D^{2} v\right]}-\frac{\psi^{\prime}}{k+\psi^{\prime \prime}|D v|^{2}} .
$$

One only needs to choose $\psi$ so that the expression above is concave in $v$. Evidently $\psi(v)=v^{2}$ qualifies. We state for completeness

Theorem 2.3. Any solution of a constant coefficient equation of the form (2) satisfying a zero Dirichlet condition on a convex domain is 1/2-concave. 


\section{The Mean Curvature Equation}

We now make some brief observations on why the two concavity maximum principle arguments of the previous section do not apply directly to the equation of constant mean curvature. Roughly speaking, we obtained Theorem 2.1 by supplying "enough convexity" to make the mapping

$$
(v, A) \mapsto \psi^{\prime}(v) \operatorname{tr}\left[a(v) A^{-1}\right]
$$

concave; see (14). This was accomplished by choosing $\psi(v)=v^{2}$ and multiplying the equation by $v$. The resulting $v$ dependence separated naturally into two terms. One was linear and we showed that the other was jointly concave much like $(v, \alpha) \mapsto$ $v^{2} / \alpha$.

A similar situation occurs if we replace $a(v)$ by $a /\left(1+\psi^{\prime 2}|D v|^{2}\right)^{3 / 2}$. Trying again the choice $\psi(v)=v^{2}$ and multiplying by $v$, one term is is again positive linear. The other term, however, looks like

$$
\frac{v^{2}}{\left(1+4 v^{2}|D v|^{2}\right)^{3 / 2}} \operatorname{tr}\left[a A^{-1}\right] .
$$

It is clear that the square root of the leading factor is not convex. Since the coefficient matrix is just $\left(\delta_{i j}\right)$ when $|D u|=0$, this suggests that one does not have "enough convexity" to make the mapping concave. This point can be made rigorous. On the other hand, multiplying by an additional factor, say a power of $v$, to overcome this problem will destroy the concavity of the linear term:

$$
(v, A) \mapsto \frac{v^{2+\epsilon}}{\left(1+4 v^{2}|D v|^{2}\right)^{3 / 2}} \operatorname{tr}\left[a A^{-1}\right]-2 H v^{1+\epsilon} .
$$

Multiplying the equation by $\left(1+\psi^{\prime 2}|D v|^{2}\right)^{3 / 2}$ clearly has a similar effect. We have not given a formal proof that no choice of $\psi$ leads to a $\psi$-adequate operator, but simply supplying factors as in the proof of Theorem 2.1 leads to the unhappy balance described above. It should be emphasized that this difficulty results exactly from the presence of the scaling $\left(1+\psi^{\prime 2}|D v|^{2}\right)^{-3 / 2}$. Consequently, exactly the same difficulty is encountered in connection with equation (13). In view of Theorem 2.2, one is inclined to view the reciprocal Laplacian argument as a "stronger" concavity maximum principle. Heuristically, it should provide quasiconcavity for constant mean curvature graphs with small mean curvature, but another difficulty arises in practice.

The argument used to obtain Theorem 2.2 relies critically upon the ability to separate the Hessian dependence from the $v$ dependence. This observation is made precise by Lemma 1.2. In fact, if one considers any map

$$
(v, A) \mapsto \frac{1}{\operatorname{tr}\left[a A^{-1}\right]}
$$


along the subspace $\{A=\alpha I\}$, then one is led to

$$
(v, A) \mapsto \frac{\alpha}{\operatorname{tr} a},
$$

which is concave only when $a$ is independent of $v$. Since such separation is evidently impossible for the mean curvature operator, it is not immediately clear how to proceed.

\section{ANOTHER APPROACH}

Continuing our discussion of the $\mathrm{CMC}$ equation, it is well known that positive solutions exist as graphs for $H$ in some interval $\left[-H_{\max }, 0\right]$, and that there are parametric CMC surfaces $\mathcal{S}=\mathcal{S}_{H}$, in the same half space as the graphs and with $\partial \mathcal{S}=\partial \Omega$, that naturally extend the family of graph solutions. See $[14,8,11$, $16,12]$. We now describe an extension of the quasiconcavity conjecture to these solutions.

We first wish to define a general quasiconcave envelope of a surface $\mathcal{S}$. Roughly speaking, this is a surface $\Sigma$ that is (i) ruled in a particular way and (ii) is tangent to $\mathcal{S}$ along its boundary. We now make this notion precise. Let $\mathcal{S}$ be a smooth surface in $\mathbb{R}^{3}$ and $\mathcal{P}=\left\{\Pi_{\alpha}\right\}$ a foliation of $\mathbb{R}^{3}$ by parallel planes. Given a point $p \in \mathcal{S}$, one can express $\mathcal{S}$ locally near $p$ as the graph of a function $u$ on $T_{p} \mathcal{S}$. We will always call such a function $u$, though the point $p$ in question will be dictated by context.

Definition 2. An embedded, connected $C^{0}$ surface $\Sigma$ is called a quasiconcave envelope on $\mathcal{S}$ with respect to $\mathcal{P}$ if:

(i) For each $\alpha$, one finds $\Sigma \cap \Pi_{\alpha}$ is a (possibly empty) collection of disjoint line segments with endpoints in $\mathcal{S}$.

(ii) $\partial \Sigma \subset \mathcal{S}$ and for each $p \in \partial \Sigma$, one has that $\Sigma$ is the graph of a function $v$ locally near $p$ on some subset $\operatorname{Dom}(v)$ in $T_{p} \mathcal{S}$. Furthermore, one can find a smooth function $w$ such that $w \geq u, w(p)=u(p)$, and, on $\operatorname{Dom}(v)$, there holds $u \leq v \leq w$.

(iii) $\Sigma$ and a portion of $\mathcal{S}$ bound an open, connected (possibly empty) volume in $\mathbb{R}^{3}$.

A quasiconcave envelope is called transverse if $\Pi_{\alpha}$ is transverse to $\mathcal{S}$ at $p$ whenever $p \in \partial \Sigma \cap \Pi_{\alpha}$.

An envelope is called simple if $\partial \Sigma \subset \operatorname{int}(\mathcal{S})$ and the volume in condition (iii) is simply connected.

Finally, an envelope is called non-empty if the enclosed volume is non-empty.

Examples. A cylinder is an empty quasiconcave envelope on itself. 
If one takes a portion of an unduloid that contains two bulges of maximum radius $r$, then there is a section of a cylinder of radius $r$ (with boundary the circles of radius $r$ on two consecutive bulges) that is a quasiconcave envelope on the unduloid with respect to any foliation of parallel planes one of which contains the common axis of the unduloid and the cylinder. In this case, the envelope is non-empty, but neither transverse nor simple.

Given a CMC surface $\mathcal{S}$ with boundary a convex planar curve, one can take for the foliation $\mathcal{P}$ the planes parallel to the plane $\Pi$ of the boundary. If $\mathcal{S}$ lies in a half space determined by $\Pi$, then replacing each level set of $\mathcal{S}$ (with respect to $\mathcal{P}$ ) by its convex hull induces a quasiconcave envelope on $\mathcal{S}$.

In the case of graphs, the result of Sakaguchi shows that the envelope is transverse. We conjecture further that the envelope is simple. The full conjecture, of course, is that the envelope must be empty. This can clearly be extended to the CMC surfaces in a half space referenced above.

We can connect the simplicity conjecture to the full conjecture explicitly as follows:

Conjecture. There is no non-empty, simple, transverse quasiconcave envelope on a CMC surface.

We conclude with the proof of the conjecture in a very special case:

Theorem 4.1. There is no non-empty quasiconcave envelope $\Sigma$ above a CMC graph with respect to vertical planes if $H \geq 0$ and $\partial \Sigma$ is smooth and transverse to the ruling segments of $\Sigma$.

Proof. Consider any point $p \in \operatorname{int}(\Sigma)$. Denote the endpoints of the segment containing $p$ by $q_{0}$ and $q_{1}$. After a rigid motion and a homothety, we may assume that $\mathcal{P}$ is a foliation by planes $\{x=$ const. $\}, q_{0}=(0,0,0)$, and $q_{1}=(0,1, u(0,1))$. For simplicity, we assume that $\partial \Sigma$ is parameterized locally near $q_{j}$ by $\left(x, j, f_{j}(x)\right)$ where $f_{j}$ is smooth. We can then express a strip of $\Sigma$ containing $p$ as the graph of

$$
v(x, y)=(1-y) f_{0}(x)+y f_{1}(x) .
$$

It follows that the mean curvature $H_{v}$ of $\Sigma$ is given locally by

$$
2 H_{v}=\frac{\left(1+\left(f_{1}-f_{0}\right)^{2}\right)\left((1-y) f_{0}^{\prime \prime}+y f_{1}^{\prime \prime}\right)-2\left((1-y) f_{0}^{\prime}+y f_{1}^{\prime}\right)\left(f_{1}-f_{0}\right)\left(f_{1}^{\prime}-f_{0}^{\prime}\right)}{\left[1+\left((1-y) f_{0}^{\prime}+y f_{1}^{\prime}\right)^{2}+\left(f_{1}-f_{0}\right)^{2}\right]^{3 / 2}} .
$$

Evaluating at $x=0$, we obtain

$$
2 H_{v}(y)=\frac{(1-y)\left[1+f_{0}^{\prime 2}+\left(f_{1}-f_{0}\right)^{2}\right]^{3 / 2} 2 H_{v}(0)+y\left[1+f_{1}^{\prime 2}+\left(f_{1}-f_{0}\right)^{2}\right]^{3 / 2} 2 H_{v}(1)}{\left[1+\left((1-y) f_{0}^{\prime}+y f_{1}^{\prime}\right)^{2}+\left(f_{1}-f_{0}\right)^{2}\right]^{3 / 2}} .
$$


It follows from the Hopf boundary point lemma, the smoothness of $\partial \Sigma$, and condition (ii) of Definition 2 that $H_{v}(0), H_{v}(1) \geq H$. Thus,

$$
\begin{aligned}
2 H_{v}(y) & \geq 2 H\left\{\frac{(1-y)\left[1+f_{0}^{\prime 2}+\left(f_{1}-f_{0}\right)^{2}\right]^{3 / 2}+y\left[1+f_{1}^{\prime 2}+\left(f_{1}-f_{0}\right)^{2}\right]^{3 / 2}}{\left[1+\left((1-y) f_{0}^{\prime}+y f_{1}^{\prime}\right)^{2}+\left(f_{1}-f_{0}\right)^{2}\right]^{3 / 2}}\right\} \\
& \geq 2 H
\end{aligned}
$$

since the map $y \mapsto\left[1+\left((1-y) f_{0}^{\prime}+y f_{1}^{\prime}\right)^{2}+\left(f_{1}-f_{0}\right)^{2}\right]^{3 / 2}$ is convex. In particular, the mean curvature of $\Sigma$ at $p$ is greater than or equal to $H$.

A slightly more complicated argument using the transversality assumption on $\partial \Sigma$ yields the general inequality $H_{v} \geq H$ for the mean curvature of $\Sigma$.

To finish the proof, we assume the assertion of the theorem is false. Then, since the enclosed volume above $\mathcal{S}$ and below $\Sigma$ is non-empty, we can lower $\Sigma$ until it is below $\mathcal{S}$ but still touching $\mathcal{S}$ at some interior point $p$. This contradicts the maximum principle.

The assumptions on the smoothness and transversality of $\partial \Sigma$ are only technicalities that greatly simplify the proof of Theorem 4.1. A proof of this result under the assumption that $H<0$ would be fundamentally different from the one presented here, and of fundamental interest.

\section{Appendix: The Main Conjecture on a Ball}

It is well known that a CMC graph with zero boundary values on a ball $B_{a}(0)=$ $\{x:|x|<a\}$ is a spherical cap and is thus concave. We extend this to the class of equations (2).

Theorem. If $\sum a_{i j}(D u) D_{i} D_{j} u=-k<0$ on $\Omega=B_{a}(0)$ and $u_{\mid \partial \Omega}=0$, then $u$ is rotationally symmetric and concave.

Proof. As noted in the introduction, it follows from the maximum principle that $u>0$ on $\Omega$. One can therefore apply an Alexandrov reflection argument as, for example, in [17] to see that $u$ is rotationally symmetric. Thus $u(x)=\varphi(|x|)$ for some smooth $\varphi$ defined by $\varphi(r)=u\left(r e_{1}\right)$ on $[-a, a]$ where $e_{1}=(1,0 \ldots, 0)$ as before.

One finds that $\varphi$ satisfies the ordinary differential equation

$$
p_{1} \varphi^{\prime \prime}+p_{2} \frac{\varphi^{\prime}}{r}=-k, \quad-a<r<a
$$

where $p_{1}=p_{1}\left(\varphi^{\prime}\right)=a_{11}\left(\varphi^{\prime} e_{1}\right)>0$ and $p_{2}=p_{2}\left(\varphi^{\prime}\right)=\sum_{i \neq 1} a_{i i}\left(\varphi^{\prime} e_{1}\right)>0$. We then have

$$
p_{1}(0) \varphi^{\prime \prime}(0)+p_{2}(0) L=-k
$$


where $L=\lim _{r \rightarrow 0}\left[\varphi^{\prime}(r) / r\right]$. On the other hand, L'Hopital's rule provides that

$$
L=\lim _{r \rightarrow 0} \varphi^{\prime \prime}(r)=\varphi^{\prime \prime}(0) .
$$

Thus,

$$
\varphi^{\prime \prime}(0)=L=-\frac{k}{p_{1}(0)+p_{2}(0)}<0
$$

We assume by contradiction the existence of a smallest positive $r_{0}$ for which $\varphi^{\prime \prime}\left(r_{0}\right)=0$. We know then that $\varphi^{\prime}(r)<0$ for $0<r \leq r_{0}$ and $\varphi^{\prime \prime}(r)<0$ for $0 \leq r<r_{0}$. Differentiating (18) and evaluating at $r=r_{0}$, we obtain

$$
p_{1} \varphi^{\prime \prime \prime}\left(r_{0}\right)=p_{2}\left(r_{0}\right) \frac{\varphi^{\prime}\left(r_{0}\right)}{r^{2}}<0,
$$

which contradicts the sign of $\varphi^{\prime \prime}$.

\section{REFERENCES}

1. O. Alvarez, J.-M. Lasry, and P.-L. Lions, Convex viscosity solutions and state constraints, J. Math. Pures Appl. 9(3) (1997), 265-288.

2. H. Brascamp and E. Lieb, On extensioins of the Brunn-Minkowski and PrékopaLeindler theorems, including inequalities for log concave functions, and with an application to the diffusion equation, J. Functional Analysis, 22 (1976), 366-389.

3. J. T. Chen and W. H. Huang, Convexity of capillary surfaces in the outer space, Invent. Math. 67(2) (1982), 253-259.

4. M. Crandall, H. Ishii, and P-L. Lions, User's guide to viscosity solutions of second order partial differential equations, Bull. Amer. Math. Soc. 27(1) (1992), 1-67.

5. D. Gilbarg and N. S. Trudinger, Elliptic Partial Differential Equations of Second Order, Springer-Verlag, Berlin, second edition, 1983.

6. B. Kawohl, Rearrangements and Convexity of Level Sets in PDE, Number 1150 in Lecture Notes in Mathematics. Springer, Berlin, 1985.

7. A. Kennington, Power concavity and boundary value problems, Indiana U. Math. J. 34(3) (1985), 687-704.

8. M. Koiso, On the uniqueness for hypersurfaces with constant mean curvature in $\mathbb{R}^{n+1}$ bounded by a round ( $n-1)$-sphere, In The Problem of Plateau, pages 129-137, World Sci., 1992.

9. N. Korevaar, Capillary surface convexity above convex domains. Indiana U. Math. J. 32 (1983), 73-81.

10. N. Korevaar, Convex solutions to nonlinear elliptic and parabolic boundary value problems, Indiana U. Math. J. 32(4) (1983), 606-614. 
11. R. Lopez, On uniqueness of constant mean curvature surfaces with planar boundary. In New Developments in differential Geometry, Budapest 1996, pages 235-242, Klewer, 1999.

12. J. McCuan, A generalized height estimate for $H$-graphs, Serrin's corner lemma, and applications to a conjecture of Rosenberg. In Minicourses of the Japan American Mathematics Institute, Johns Hopkins University, 1999, to appear.

13. J. McCuan, Continua of $H$-graphs: convexity and isoperimetric stability, Calc. Var. Partial Differential Equations, 9(4) (1999), 297-325.

14. A. Ros and H. Rosenberg, Constant mean curvature surfaces in a half-space of $\mathbb{R}^{3}$ with boundary in the boundary of the half-space, J. Differential Geom. 44(4) (1996), 807-817.

15. S. Sakaguchi, Uniqueness of critical point of the solution to the prescribed constant mean curvature equation over convex domain in $\mathbb{R}^{2}$. In Recent Topics in Nonlinear PDE IV, Kyoto, 1988, volume 10 of Lecture Notes in Num. Appl. Anal. pages 129-151, 1989.

16. B. Semmler, The topology of large $H$-surfaces bounded by a convex curve, Ann. Sci. École Norm. Sup. (4), 33(3) (2000), 345-359.

17. H. Wente, The symmetry of sessile and pendent drops, Pacific J. Math. 88(2) (1980), 387-397.

John McCuan

School of Mathematics

Georgia Institute of Technology

Atlanta, GA 30332-0160

U.S.A.

E-mail: mccuan@math.gatech.edu 